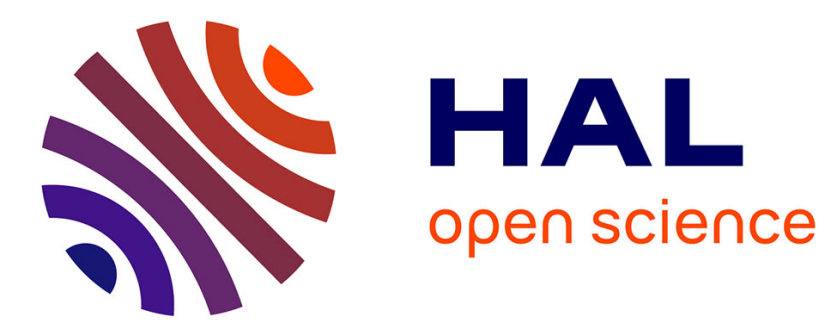

\title{
Quand les entreprises s'emparent de la mort numérique, qui sont les consommateurs potentiels ?
}

Samuel Guillemot, Andréa Gourmelen

\section{To cite this version:}

Samuel Guillemot, Andréa Gourmelen. Quand les entreprises s'emparent de la mort numérique, qui sont les consommateurs potentiels ?. Revue Française de Gestion, 2017, 43 (262), pp.123 - 145. 10.3166/rfg.2017.00114. hal-01676282

\section{HAL Id: hal-01676282 \\ https://hal.umontpellier.fr/hal-01676282}

Submitted on 4 May 2021

HAL is a multi-disciplinary open access archive for the deposit and dissemination of scientific research documents, whether they are published or not. The documents may come from teaching and research institutions in France or abroad, or from public or private research centers.
L'archive ouverte pluridisciplinaire HAL, est destinée au dépôt et à la diffusion de documents scientifiques de niveau recherche, publiés ou non, émanant des établissements d'enseignement et de recherche français ou étrangers, des laboratoires publics ou privés. 


\title{
Quand les entreprises s'emparent de la mort numérique, qui sont les consommateurs potentiels?
}

\author{
Samuel GUILLEMOT, Univ Brest, LEGO, Quimper, France \\ Andréa GOURMELEN, Univ Montpellier, MRM, Montpellier, France
}

\begin{abstract}
Résumé. Le flou juridique autour de la gestion des traces numériques des défunts (profils de réseaux sociaux, documents ou photos déposés sur des clouds) amène des entreprises à inciter les internautes à anticiper leur mort numérique. Cependant, la pratique se développe difficilement. Cet articlel identifie cinq profils d'internautes qui se distinguent vis-à-vis de leur intention de préparer leur mort numérique sur la base de trois variables : l'orientation temporelle, l'attachement aux contenus numériques et les motivations à transmettre la mémoire. Des pistes d'actions marketing sont suggérées afin de développer ces pratiques..
\end{abstract}

Mots clés : Mort numérique / préparation à la mort / motivations agentiques et communales / extended-self

TO CITE: GUILLEMOT S. AND GOURMELEN, A. (2017), QUAND LES ENTREPRISES S'EMPARENT DE LA MORT NUMERIQUE, QUI SONT LES CONSOMMATEURS POTENTIELS?, REVUE FRANÇAISE DE GESTION, 43 (262), 123-145

«Avez-vous conscience de la valeur de votre héritage numérique?». A travers cette question, Edeneo, site spécialisé dans les coffres-forts numériques testamentaires, tente de sensibiliser les internautes à la préparation de leur mort numérique. Aujourd'hui, un individu laisse davantage de traces numériques que de traces physiques de lui-même. Tout au long de sa vie, il crée et diffuse des contenus à son image (photos, vidéos, e-mails, commentaires, données personnelles, etc.). Pour la seule année 2010, chaque internaute aurait produit en moyenne 1200 mégabits de données, soit l'équivalent de 602 photos (5 mégapixels) ou encore de 120000 pages de traitement de texte (Caroll et Romano, 2010). Ces contenus numériques sont le plus souvent stockés en ligne sur des serveurs (cloud) et/ou partagés sur les réseaux sociaux. Or, qu'arrive-t-il à ces contenus une fois la personne décédée ?

Les données informatiques sont soumises à un régime spécifique qui exclut leur transmission suite à un décès (loi $\mathrm{n}^{\circ} 78-17$ du 6 janvier 1978 relative à l'informatique, aux fichiers et aux libertés). La «loi numérique 2016 » vise néanmoins à établir pour chacun le droit d'exprimer sa volonté sur le devenir de ses contenus numériques en ligne après sa mort. Les enjeux concernant la préparation de sa mort numérique sont bien réels pour les entreprises. Si rien n'est fait, à moyen terme, le nombre de profils de réseaux sociaux appartenant à des personnes décédées excédera celui de personnes vivantes (Zeller, 2012).

Les acteurs du web ont d'ores et déjà mis en place des solutions pour faire face au décès de leurs utilisateurs, en les incitant à organiser le devenir de leurs contenus numériques après leur mort. Ils proposent d'effectuer des démarches en ligne pour formuler des directives anticipées (désigner des héritiers pour leur fournir des mots de passe de comptes ou encore des consignes pour savoir quels profils supprimer, quels autres conserver) et/ou réorganiser leurs traces en ligne (rédaction de carnets de vie numériques, par exemple).

Néanmoins, ces nouveaux services ne rencontrent pas le succès escompté et beaucoup d'entreprises cessent leurs activités. L'objectif de cet article est de décrire les utilisateurs potentiels en fournissant aux entreprises une typologie permettant de segmenter le marché et ainsi, d'adapter leurs offres de services. L'article débute par une présentation du marché de la préparation à la mort numérique. Il se prolonge par une revue de la littérature, essentiellement 
centrée sur la mort physique en comportement du consommateur, ce qui permet d'éclairer la compréhension du comportement des internautes au regard de ces questions de mort numérique. La typologie en cinq profils d'internautes est ensuite présentée. Celle-ci est élaborée à partir d'une étude quantitative réalisée auprès de 280 individus. L'article se termine par une discussion des résultats, présentant des préconisations managériales.

\section{LE MARCHE DE LA PREPARATION A LA MORT NUMERIQUE}

Il existe divers services qui entendent accompagner les pratiques liées à la préparation de son décès ${ }^{1}$. Deux types d'acteurs peuvent-être identifiés sur ce marché: les acteurs institutionnels du web ayant un discours pragmatique sur la mort et les entreprises spécialisées qui vendent des services de préparation à la mort et emploient pour cela un discours sacré sur la mort.

\section{Les acteurs institutionnels}

Le cabinet FaberNovel $^{2}$ estime que $55 \%$ de la vie numérique (e-mail, e-commerce, musique, vidéo, réseau social) d'un internaute moyen sont passés sur les quatre plates-formes des GAFA (Google, Apple, Facebook et Amazon). Ces quatre acteurs institutionnels sont les premiers concernés par le décès de leurs utilisateurs. Ils réagissent de manière plus ou moins engagée en incitant leurs utilisateurs à anticiper leur mort numérique. Avec son service Google Inactive Account Manager, Google responsabilise les utilisateurs et leur offre la possibilité de désigner un tiers de confiance, à qui sera transmis les identifiants et mots de passe du compte Google, après une période d'inactivité définie à l'avance par l'utilisateur. Facebook a adopté une position similaire depuis 2015, puisqu'il propose à l'utilisateur de désigner un contact légataire. En revanche, Apple précise dans ses conditions d'utilisation que les droits associés au compte ne sont pas transférables suite au décès de l'utilisateur. Ce sont des services censés faciliter l'usage des services cœur de métier. En raisonnant en termes de carte de services, on peut les considérer comme des services périphériques, susceptibles de faire la différence par rapport aux concurrents.

Ces acteurs institutionnels présentent leurs services en employant un discours neutre centré sur une chose à faire. Nous observons néanmoins un certain déni de la mort. En effet, les prestataires évoquent l'inactivité du compte ou désignent par compte inactif, le compte d'un défunt. Par exemple, «N'oubliez pas que l'utilisateur inactif a peut-être choisi de supprimer automatiquement son compte si celui-ci a été inactif pendant une durée définie. Il peut également avoir désigné plusieurs personnes de confiance»(Site de Google - rubrique Inactive Account Manager).

\section{Les acteurs spécialistes}

Depuis une dizaine d'années, est apparue une autre forme d'acteur sur le marché de la préparation de la mort numérique. Il s'agit de prestataires spécialisés comme E-mylife ou Edeneo qui proposent des offres de services très complètes telles que la location d'un coffrefort numérique testamentaire, dans lequel l'individu dépose les mots de passe de tous ses comptes en ligne et/ou divers documents (photos, livre de vie pdf, vidéos) à destination de ses

\footnotetext{
${ }^{1}$ Cet article se focalise sur les services concernant sa propre mort et n'évoque pas les services concernant la réaction à la disparition d'un proche. Ces services (comme le cimetière virtuel) s'adressent à ceux qui restent après un décès et permettent de rendre hommage à un proche décédé. Ils relèvent ainsi d'une autre problématique liée aux pratiques de deuil et non de préparation de sa mort numérique.

${ }^{2}$ http://www.fabernovel.com/fr/gafa/
} 
héritiers. La différence avec ce que proposent Google et Facebook est que ces prestataires élargissent le service à l'ensemble des comptes en ligne possédés par un individu. Ce dernier peut alors indiquer des instructions relatives à chaque compte, pour en transmettre certains et en effacer d'autres.

D'autres sites web s'adressent à des individus souhaitant transmettre quelque chose de personnalisé, à l'image d'une biographie en ligne, à leurs descendants. Ces entreprises proposent de créer un document unique à partir d'éléments numériques existants en ligne ou sur l'ordinateur des individus. Par exemple, le site La Vie D'Après propose : "Avant et après, la vie, toujours. Créez un espace pour votre héritage numérique. Sauvegardez et transmettez vos souvenirs ». Ces prestataires parlent de la mort comme d'une étape logique de la vie. Ils tentent de rassurer les utilisateurs quant à leur démarche. Les discours sont centrés sur le futur, le fait de laisser aux générations suivantes un héritage sentimental, une trace de soi, ou encore d'atteindre une vie éternelle digitale. Les services concernés accompagnent des pratiques individuelles et collectives, en lien (ou non) avec les croyances. Ils entrent ainsi dans le domaine du sacré et de l'immortalité symbolique.

Le tableau 1 présente de manière synthétique les caractéristiques principales des offres de services identifiées sur le marché. Celles-ci peuvent être classées en trois catégories selon leur contenu: les services de transmission de mots de passe ou instructions (proposés par les acteurs institutionnels comme par les spécialistes), les coffres-forts numériques testamentaires (proposés uniquement par les spécialistes) et les services créatifs d'organisation des traces en ligne (proposés uniquement par les spécialistes). 
Tableau 1 : Synthèse des principales offres disponibles sur le marché.

\begin{tabular}{|c|c|c|c|c|}
\hline Prestataire & Prestation de service & Tarif & Durée & Garanties proposées \\
\hline \multicolumn{5}{|c|}{ Les services de transmission de mots de passe ou instructions } \\
\hline $\begin{array}{l}\text { Google (Inactive } \\
\text { account manager) }\end{array}$ & $\begin{array}{l}\text { Transmission de données liées au compte Google } \\
\text { préalablement sélectionnées et/ou d'un message en cas } \\
\text { d'inactivité sur son compte Google }\end{array}$ & Gratuit & A vie & $\begin{array}{l}\text { Message à un contact préalablement désigné en cas de compte inactif. Transmission des } \\
\text { données préalablement sélectionnées (ex : Gmail, Google Drive) par un lien de téléchargement } \\
\text { envoyé au destinataire }\end{array}$ \\
\hline $\begin{array}{l}\text { Facebook (contact } \\
\text { légataire) }\end{array}$ & $\begin{array}{l}\text { Désignation d'un destinataire pour gérer son compte } \\
\text { Facebook après son décès ou demander la suppression } \\
\text { du compte suite à son décès }\end{array}$ & Gratuit & A vie & $\begin{array}{l}\text { Choix d'un destinataire et d'actions pour ce destinataire (gestion du compte, téléchargement } \\
\text { des données) } \\
\text { Ou choix de suppression du compte post-mortem (automatiquement sans désignation de } \\
\text { contact légataire) }\end{array}$ \\
\hline E my life & $\begin{array}{l}\text { Fermeture ou volonté particulière sur tous comptes } \\
\text { Internet (ex : boîtes mails, Facebook, blogs, PayPal) }\end{array}$ & $\begin{array}{l}35 € \\
\text { compte }\end{array}$ & A vie & $\begin{array}{l}\text { Formalités liées à la clôture de certains comptes ou transmission post-mortem gérées par le } \\
\text { prestataire }\end{array}$ \\
\hline \multicolumn{5}{|c|}{ Les coffres-forts numériques testamentaires } \\
\hline \multirow[t]{3}{*}{ Edeneo } & \multirow{3}{*}{$\begin{array}{l}\text { Coffre-fort numérique testamentaire élargi : dépôt de } \\
\text { tous fichiers, tous formats, y compris identifiants et } \\
\text { mots de passe }\end{array}$} & Gratuit & $\begin{array}{l}\text { Non } \\
\text { précisée }\end{array}$ & $\begin{array}{l}3 \text { comptes internet, } 1 \text { message et } 1 \text { destinataire (formule qui semble être en cours de } \\
\text { suppression) }\end{array}$ \\
\hline & & $29,90 €$ & 1 an & \multirow[t]{2}{*}{2 Go de données - Nombre de destinataires illimité } \\
\hline & & $299 €$ & A vie & \\
\hline \multirow[t]{4}{*}{ E my Life } & \multirow{2}{*}{$\begin{array}{l}\text { Coffre-fort numérique testamentaire centré sur les } \\
\text { messages, photos, vidéos }\end{array}$} & $28 €$ & 1 an & \multirow[t]{2}{*}{3 Go de données, points fidélité, choix du destinataire de chaque document } \\
\hline & & $229 €$ & A vie & \\
\hline & \multirow[t]{2}{*}{$\begin{array}{l}\text { Extension de l'espace de stockage }\left(\begin{array}{llll}1 & \text { à } & 10 & \text { Go } \\
\text { supplémentaires) }\end{array}\right.\end{array}$} & $\begin{array}{l}8 € \text { à } 55 € / \\
\text { an }\end{array}$ & 1 an & 1 Go $(8 €)$ à 10 Go de plus $(55 €)$, points fidélité offerts \\
\hline & & $89 €$ à $749 €$ & A vie & 1 Go $(89 €)$ à 10 Go de plus $(749 €)$, points fidélité offerts \\
\hline \multirow[t]{2}{*}{ La Vie d'Après } & \multirow{2}{*}{$\begin{array}{l}\text { Coffre-fort numérique testamentaire élargi : dépôt de } \\
\text { tous fichiers, tous formats, y compris identifiants et } \\
\text { mots de passe }\end{array}$} & Gratuit & A vie & Offre Découverte : 10 Mo d'espace de stockage - 1 seul destinataire possible \\
\hline & & $20 €$ & 1 an & $\begin{array}{l}\text { Offre Sérénité : } 5 \text { Go d'espace de stockage - Nombre illimité de destinataires - Journal de vie } \\
\text { interactif }\end{array}$ \\
\hline \multicolumn{5}{|c|}{ Les services créatifs d'organisation des traces en ligne } \\
\hline E my Life & $\begin{array}{l}\text { Service complémentaire de vidéo pour les clients } \\
\text { disposant d'un « coffre-fort numérique » }\end{array}$ & $199 €$ & $\begin{array}{l}\text { Non } \\
\text { applicable }\end{array}$ & Tournage d'une vidéo à domicile, montage, dépôt dans le coffre-fort numérique de l'individu. \\
\hline Eternissim & $\begin{array}{l}\text { Rédaction d'une biographie interactive, de messages } \\
\text { post-mortem à transmettre aux proches, organisation } \\
\text { d'une médiathèque contenant des photos, vidéos, } \\
\text { musiques }\end{array}$ & $99 €$ & A vie & $\begin{array}{l}1 \text { Go d'espace de stockage } \\
\text { Choix des éléments privés, publics et des destinataires }\end{array}$ \\
\hline La Vie d'Après & $\begin{array}{l}\text { Construction d'un ouvrage interactif et multimédia } \\
\text { relatif à l'histoire de sa vie }\end{array}$ & $20 €$ & 1 an & $\begin{array}{l}\text { Option comprise dans la formule « Sérénité » avec le coffre-fort numérique associé : } 5 \text { Go au } \\
\text { total (coffre-fort }+ \text { ouvrage) } \\
\text { Nombre illimité de chapitres }\end{array}$ \\
\hline $\begin{array}{l}\text { Mon album de vie } \\
\text { (lié à une fondation } \\
\text { d'utilité publique) }\end{array}$ & $\begin{array}{l}\text { Rédaction d'une biographie interactive, de messages } \\
\text { post-mortem à transmettre aux proches, contenant des } \\
\text { photos, vidéos, musiques, lettres... }\end{array}$ & Gratuit & A vie & $\begin{array}{l}\text { Pas de précision quant au nombre de destinataires et le poids maximum de données autorisées. } \\
\text { Format livre interactif }\end{array}$ \\
\hline \multirow[t]{2}{*}{ Stone story } & \multirow[t]{2}{*}{ Construction d'une histoire de vie } & Gratuit & A vie & $\begin{array}{l}\text { Rubriques limitées à la nécrologie, à une page de condoléances et un carnet d'adresses de } \\
\text { proches }\end{array}$ \\
\hline & & $95,90 €$ & 5 ans & $\begin{array}{l}\text { Rubriques enrichies (fonctions : biographie, photos, vidéos, Code } \mathrm{QR} \text { à apposer sur un objet } \\
\text { réel, comme la pierre tombale par exemple). Publication de «l'histoire de vie » uniquement au } \\
\text { décès de l'individu }\end{array}$ \\
\hline
\end{tabular}


Nous n'avons pas accès aux données des acteurs sur le nombre d'utilisateurs de ces services. Néanmoins, il semblerait que certains d'entre eux ne rencontrent pas le succès attendu. En effet, de nombreux acteurs spécialistes ont cessé leur activité après quelques mois ou années d'existence comme lab109, Memolane, Masaga, For U Forever ou encore Memory-life. D'autres, à l'image de Biobble, ne se développent pas ou ne sont plus actualisés. Nous pouvons alors nous demander si ces services sont adaptés. Dans quelle mesure les utilisateurs envisagent-ils de préparer leur mort numérique ? La littérature apporte quelques éléments de réponse à ces questions.

\section{LES VARIABLES SUSCEPTIBLES D'AFFECTER LA PREPARATION DE SA MORT NUMERIQUE}

La tendance humaine à se focaliser sur sa propre mort est bien présente dans la littérature en sciences de gestion, notamment en comportement du consommateur (Urien, 2003). Les recherches se limitent bien souvent à l'étude de la mort physique. En ce sens, elles traitent de l'anticipation de sa mort et des actions entreprises pour gérer son anxiété (Urien et Guiot, 2007). En croisant ce cadre avec les caractéristiques des possessions numériques (Belk, 2013), trois variables apparaissent susceptibles de jouer un rôle important pour expliquer les comportements de préparation de sa mort dans un univers numérique : l'orientation temporelle, l'attachement aux contenus numériques et les motivations à transmettre la mémoire. Ces variables sont néanmoins à mettre en parallèle avec un certain nombre de freins liés aux contours flous et incertains de son identité numérique.

\section{L'orientation temporelle}

Si la plupart des recherches sur la préparation de sa mort concerne les «seniors », les chercheurs reconnaissent que ce n'est pas tant l'âge qui est déterminant mais la perception subjective du temps, notamment du temps qui reste à vivre. Ainsi, Malas (2012) constate que les individus commencent à épargner pour autrui (transfert de patrimoine à leurs enfants) avant la cinquantaine. Si les actions concrètes concernant la planification de l'héritage se développent dans la dernière partie de la vie, c'est parce que le patrimoine s'accumule et que l'individu se rapproche de sa mort. Néanmoins, les individus peuvent y penser beaucoup plus tôt dans le cycle de vie. D'ailleurs, Waagstein (2014) montre que les jeunes internautes sont sensibles aux questions relatives à la mort numérique. Ce n'est pas tant l'âge, mais plutôt l'orientation temporelle - la focalisation de l'individu sur le passé, le présent et/ou le futur (Usunier et Valette-Florence, 2007) - qui apparaît importante pour expliquer les intentions de préparer sa mort. Autrement dit, un individu orienté futur aura davantage tendance à s'interroger sur l'avenir de ses traces en ligne et de ses contenus numériques qu'un individu orienté présent.

\section{L'attachement aux contenus numériques}

Dès lors que l'individu est préoccupé par le futur, il va pouvoir réfléchir au sort de ses possessions qui ont une valeur à ses yeux. Outre le cas particulier de la perpétuation du patrimoine financier (patrimoine immobilier, succession, donation, etc.), les individus sont particulièrement préoccupés par le sort de leur patrimoine symbolique qui se matérialise dans des objets spéciaux qui servent de véhicule à l'histoire familiale, individuelle et collective (Curasi et al., 2004). Ces objets, qui emprisonnent le passé, sont indexés à des moments de vie ou à des personnes : cela peut être des photos, des souvenirs d'un évènement, ou encore un 
objet ayant appartenu à un parent. Ce lien existe aussi dans l'univers numérique. Les individus peuvent s'attacher à leurs contenus numériques (photos, profils de réseaux sociaux, fichiers) en raison du temps et des efforts consacrés à les faire évoluer, les préserver et/ou les personnaliser (Denegri-Knott et al., 2012) ; ou parce qu'ils emprisonnent une partie des souvenirs ou de la personnalité d'un individu, finissant par représenter une partie de lui-même (extended-self, Belk, 2013). Cependant, l'attachement serait moins fort que pour les objets physiques, en raison du caractère réplicable et intangible du numérique (Belk, 2013). Les jeunes générations, entrées dans le monde connecté à un âge plus précoce que les générations qui les précèdent, seraient cependant plus promptes à considérer les contenus numériques comme naturels et à y être attachés (Cushing, 2012).

\section{Les motivations à transmettre la mémoire}

En dernier ressort, si l'individu est préoccupé par le futur des objets qui emprisonnent une partie de sa mémoire, il va être motivé pour les transmettre. Plusieurs tactiques possibles vont se distinguer, en fonction de la nature agentique ou communale des motivations à transmettre la mémoire (Guillemot et Urien, 2016). Les motivations agentiques font référence à la volonté de promouvoir sa propre personne à travers la transmission d'un objet porteur de mémoire. Les individus espèrent ne pas être oubliés après leur mort, ils cherchent alors à contrôler l'image qu'ils laisseront d'eux. Les motivations communales font, quant à elles, référence aux actions motivées par le fait de préserver un sens commun et de laisser des traces qui pourront être utiles et servir les générations futures. Ces motivations apparaissent d'autant plus importantes dans un univers numérique : s'il est relativement aisé de contrôler la destinée de ses traces physiques (destruction de documents, transmission à une personne, etc.), cela est moins évident - voire impossible - avec les traces en ligne. En effet, les représentations de soi sont intangibles, éparpillées (Belk, 2013) à travers les différents comptes en ligne, les photos postées sur les réseaux sociaux, les commentaires laissés sur la toile, ou encore les documents personnels stockés dans les clouds.

\section{Les freins liés au contexte particulier de la mort numérique}

Si les individus sont sensibilisés aux problèmes posés par la mort numérique à travers la mort de leurs proches ou de personnalités (Waagstein, 2014), peu passent à l'action en prenant des dispositions pour anticiper leur propre décès. Plusieurs arguments peuvent être avancés pour l'expliquer. Tout d'abord, l'évitement est un mécanisme d'adaptation pour gérer le stress provoqué par le fait de penser à sa propre mort (Urien, 2003). Cette inaction est une problématique partagée par les acteurs traditionnels liés à la mort (pompes funèbres, notaires pour la transmission du patrimoine, par exemple).

Ensuite, cette difficulté est accentuée par les contours mouvants de l'identité numérique, ce qui rend difficile l'évaluation de son empreinte numérique. En effet, l'identité numérique est définie comme «l'ensemble des représentations numériques des traits sociaux et personnels d'un individu qui sont exprimés au travers des environnements numériques » (Sohier et Brée, 2016, p.2). L'individu n'en a pas totalement le contrôle car elle est le fruit d'une interaction entre les stratégies identitaires des individus et les algorithmes interactifs des plateformes web (Georges, 2014). De plus, ces représentations de soi sont largement partagées et co-construites avec les autres personnes connectées, qui vont laisser leurs propres commentaires sur les photos d'un individu, par exemple.

Enfin, les individus sont globalement réticents à confier leurs données numériques à des entreprises privées, qui s'inscrivent dans une logique économique et qui peuvent cesser leur 
activité à tout moment. Ainsi, quelques individus développent des actions manuelles, qui viennent concurrencer les services existants : principalement la transmission de mots de passe oralement ou sur un papier en cas de besoin, ou l'impression des photos numériques qui leur tiennent le plus à cœur (Guillemot et Gourmelen, 2014).

\section{UNE TYPOLOGIE D'INDIVIDUS SELON LEURS INTENTIONS DE PREPARER LEUR MORT NUMERIQUE}

Les services de préparation à la mort numérique répondent à un impératif de la part des prestataires, mais ceux-ci ne semblent pas rencontrer le succès. Pourtant, la théorie montre que les internautes peuvent être préoccupés par ces questions de mort numérique à partir du moment où ils ont tendance à se projeter dans le futur, à être attachés à leurs contenus numériques et avoir des motivations à transmettre la mémoire de nature agentique et/ou communale. Compte-tenu du caractère récent du marché, une démarche descriptive est privilégiée. Dans une perspective de découverte, nous cherchons à décrire les attitudes et intérêts des internautes envers la préparation de leur mort numérique, en les classant dans des groupes qui refléteront les grandes tendances. Ainsi, cette partie présente une typologie d'internautes en cinq profils sur la base de ces variables. Elle débute par une présentation du design de l'étude avec les modalités de recrutement des répondants, la nature des questions posées et les traitements statistiques effectués, puis s'achève par une présentation des résultats.

\section{Recrutement des répondants}

280 internautes ont été interrogés via un questionnaire auto-administré en ligne, visant à déterminer leurs intentions de préparer leur mort numérique. Les répondants ont été recrutés en ligne ; le lien vers le questionnaire ayant été posté sur des réseaux sociaux, des forums et également envoyé via des listes de diffusion pour encourager la diversité des répondants et l'effet boule de neige. Les conditions de participation étaient d'avoir au minimum 18 ans et d'être actif sur le web. En effet, les conditions d'utilisation des sites évoqués dans le tableau 1 mettent généralement en avant la nécessité d'avoir au minimum 18 ans pour souscrire à une formule. C'est également l'âge auquel il est possible de désigner un contact légataire sur Facebook (les utilisateurs plus jeunes n'ont pas cette possibilité). A titre de comparaison, la moyenne d'âge du réseau social le plus utilisé - Facebook - est de 41 ans $^{3}$ et celui de notre échantillon est de 34,5 ans. Au final, notre échantillon apparaît varié en termes de genre, d'âge et d'activités sur le web (tableau 2).

\section{Structure du questionnaire}

Pour refléter la diversité des contenus numériques auxquels un individu peut être attaché tout en évitant un questionnaire trop répétitif, deux éléments numériques potentiellement transmissibles ont été sélectionnés : les profils de réseaux sociaux et les contenus numériques stockés dans un cloud. Les autres traces numériques sur le net (commentaires, etc.) ont été écartées car il est difficile pour les personnes de se rendre compte de l'ampleur de celles-ci.

Le questionnaire est divisé en quatre parties : une première partie pour mesurer et évaluer l'activité sur le web des internautes (nombre de comptes, ancienneté, usages) ainsi que leur

\footnotetext{
${ }^{3}$ https://www.forrester.com
} 
degré d'attachement à leur profil de réseau social et aux contenus de leur cloud ${ }^{4}$. La deuxième partie est focalisée sur la mesure des motivations à transmettre la mémoire. La troisième partie mesure les intentions d'utiliser les services de préparation à la mort numérique. Ainsi, trois items représentant les services spécialisés identifiés dans la première partie, ainsi que quatre items représentant des alternatives impliquant ou non un passage dans le monde physique, ont été formulés (fonctionnalités biographiques des réseaux sociaux, notaire, impression, document papier) (tableau 3).

Tableau 2 : Caractéristiques de l'échantillon $(\mathrm{n}=280)$

\begin{tabular}{llll}
\hline Variables sociodémographiques & & Variables comportementales \\
\hline Age & & Comportement sur les réseaux sociaux & \\
$18-25$ ans & $26,2 \%$ & Fréquence de consultation & \\
$26-50$ ans & $59,7 \%$ & Quotidienne & $76,9 \%$ \\
51 ans et plus & $14,1 \%$ & Hebdomadaire & $12,1 \%$ \\
Genre & & Moins souvent & $11 \%$ \\
Homme & $42,1 \%$ & Usages & $69,2 \%$ \\
Femme & $56,9 \%$ & Commenter l'actualité & $35,6 \%$ \\
CSP & & Discuter en ligne (conversations privées) & $14,9 \%$ \\
Etudiant & $21,1 \%$ & Publier du contenu sur soi & $17,1 \%$ \\
Actif en emploi & $74,6 \%$ & Publier du contenu général & \\
Actif sans emploi & $3,2 \%$ & & \\
Retraité & $1,1 \%$ & Comportement sur les clouds & \\
Situation familiale & & Usages & $64,5 \%$ \\
Célibataire & $39,4 \%$ & Stocker des photos/vidéos personnelles & \\
En couple & $55,2 \%$ & Stocker d'autres documents personnels & $63,4 \%$ \\
Autres (divorcé, veuf) & $5,4 \%$ & Stocker de la musique, des films & $17,5 \%$ \\
Niveau d'études & & Stocker des documents professionnels & $65,9 \%$ \\
$<$ Niveau bac & $24,6 \%$ & & \\
Niveau bac & $26,5 \%$ & & \\
$>$ Bac & $48,9 \%$ & & \\
\hline
\end{tabular}

Tableau 3. La mesure des intentions d'utiliser des services de préparation à la mort numérique

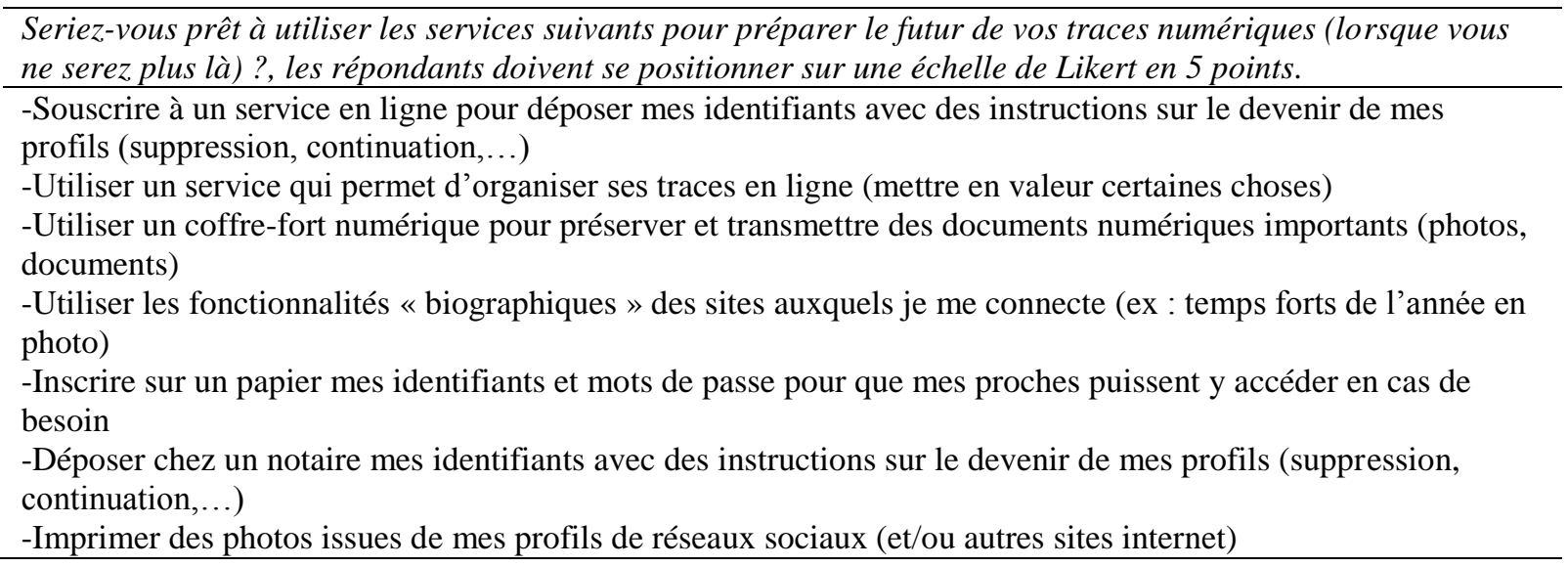

Une attention toute particulière a été portée à la façon d'évoquer la mort des répondants. En effet, une situation de saillance de mort trop prononcée peut provoquer des émotions négatives conduisant à l'abandon du questionnaire ou à des réponses biaisées. C'est pourquoi

\footnotetext{
${ }^{4}$ Les répondants étaient invités à penser à un réseau social et à un cloud en particulier. Le réseau social le plus cité par les répondants est Facebook (86.6\%). Pour les cloud, il s'agit de Dropbox (45.5\%), suivi de Google Drive (26.8\%).
} 
la notion a été introduite progressivement; d'abord de manière indirecte (« vos traces numériques dans 50 et 100 ans »), puis de manière directe («lorsque vous ne serez plus là ») pour les mesures d'intention d'utiliser les services. Le questionnaire se termine par des questions visant à décrire l'échantillon en termes sociodémographiques. L'échelle d'orientation temporelle a été introduite ici. De même, une mesure de l'expertise perçue relative à l'utilisation d'internet a été ajoutée, car elle peut jouer un rôle dans le passage des intentions en actions (tableau 4).

Tableau 4. Les échelles de mesure (format Likert 5 points)

\begin{tabular}{|c|c|c|c|}
\hline Mesure & $\begin{array}{l}\text { Nombre } \\
\text { d'items }\end{array}$ & Exemples & $\begin{array}{c}\text { Fiabilité } \\
(\alpha)\end{array}$ \\
\hline \multicolumn{4}{|l|}{$\begin{array}{l}\text { Attachement aux contenus } \\
\text { numériques / Items formulés sur la } \\
\text { base de l'Extended-self(Belk, 2013) }\end{array}$} \\
\hline -Profils des réseaux sociaux & 3 & $\begin{array}{l}\text { «Si mon profil devait être supprimé, je serais } \\
\text { triste de le perdre» }\end{array}$ & 0,74 \\
\hline -Contenu des clouds & 3 & $\begin{array}{l}\text { «Je peux dire que le contenu de ce cloud reflète } \\
\text { une partie de ma vie» }\end{array}$ & 0,82 \\
\hline \multicolumn{4}{|l|}{$\begin{array}{l}\text { Motivations à transmettre la } \\
\text { mémoire / Echelle Motivations au } \\
\text { Récit de Vie adaptée au numérique } \\
\text { (Guillemot et Urien, 2016) }\end{array}$} \\
\hline -Motivations agentiques & 7 & $\begin{array}{l}\text { «Il est important de préserver les traces de ce } \\
\text { qui a été publié en ligne car cela permettra de } \\
\text { montrer ce que j'ai pu faire d'intéressant dans la } \\
\text { vie » }\end{array}$ & 0.90 \\
\hline -Motivations communales & 7 & $\begin{array}{l}\text { «Il est important de préserver les traces de ce qui } \\
\text { a été publié en ligne car cela permettra d'aider la } \\
\text { famille à des fins généalogiques » }\end{array}$ & 0.91 \\
\hline $\begin{array}{l}\text { Orientation temporelle futur / } \\
\text { Usunier et Valette-Florence (2007) }\end{array}$ & 4 & $\begin{array}{l}\text { «Je passe du temps à penser à ce que mon futur } \\
\text { pourrait être » }\end{array}$ & 0.86 \\
\hline $\begin{array}{l}\text { Expertise perçue / Bécheur et } \\
\text { Gollety (2006) }\end{array}$ & 4 & $\begin{array}{l}\text { «J'en sais plus que la moyenne des gens sur } \\
\text { internet» }\end{array}$ & 0.89 \\
\hline
\end{tabular}

\section{Analyse des résultats}

Les analyses typologiques (cf. encadré méthodologique) permettent de dégager cinq segments d'individus qui diffèrent de par leur intérêt porté aux services de préparation à la mort numérique et les raisons qui sous-tendent cet intérêt (tableau 6). 
La fiabilité et la validité des échelles de mesure ont d'abord été vérifiées à l'aide d'analyses factorielles en composantes principales (ACP). Des analyses typologiques ont ensuite été effectuées. La typologie s'est construite sur la base des 7 possibilités de préparation à la mort numérique identifiées (services et solutions alternatives des consommateurs). Une ACP réalisée sur ces 7 items révèle la présence d'un seul facteur $(\alpha=0.83)$, ce qui signifie que les consommateurs catégorisent ces services comme étant relativement homogènes (ils permettent tous la préparation de sa propre mort numérique). Afin de préserver une richesse d'analyse et compte-tenu de la perspective de découverte, il a été décidé de ne pas utiliser le score factoriel relatif aux services et ainsi de laisser les 7 items covarier (tableau 5).

Tableau 5. Matrice de corrélations des items d'intention

\begin{tabular}{lllllll}
\hline Service de & Coffre-fort & Service & Fonctions & Ecrire & Dépôt & Impression \\
transmission & numérique & créatif & biographi & sur & chez un & photos \\
mots de & testamentaire & d'organisa & ques de & papier & notaire & issues de \\
passe & & tion des & profils & identifia & d'identifia & profils \\
linstructions & & traces en & existants & nts-mots & nts-mots & \\
en ligne & & ligne & & de passe & de passe \\
\hline
\end{tabular}

\begin{tabular}{|c|c|c|c|c|c|c|c|}
\hline $\begin{array}{l}\text { Service de } \\
\text { transmission mots } \\
\text { de passe / } \\
\text { instructions en ligne }\end{array}$ & 1 & & & & & & \\
\hline $\begin{array}{l}\text { Coffre-fort } \\
\text { numérique } \\
\text { testamentaire }\end{array}$ & 0.54 & 1 & & & & & \\
\hline $\begin{array}{l}\text { Service créatif } \\
\text { d'organisation des } \\
\text { traces en ligne }\end{array}$ & 0.61 & 0.61 & 1 & & & & \\
\hline $\begin{array}{l}\text { Fonctions } \\
\text { biographiques de } \\
\text { profils existants }\end{array}$ & 0.51 & 0.47 & 0.47 & 1 & & & \\
\hline $\begin{array}{l}\text { Ecrire sur papier } \\
\text { identifiants-mots de } \\
\text { passe }\end{array}$ & 0.31 & 0.37 & 0.32 & 0.30 & 1 & & \\
\hline $\begin{array}{l}\text { Dépôt chez un } \\
\text { notaire identifiants- } \\
\text { mots de passe }\end{array}$ & 0.61 & 0.51 & 0.56 & 0.41 & 0.36 & 1 & \\
\hline $\begin{array}{l}\text { Impression de } \\
\text { photos issues de } \\
\text { profils }\end{array}$ & 0.24 & 0.28 & 0.29 & 0.53 & 0.28 & 0.31 & 1 \\
\hline
\end{tabular}

Pour limiter les risques liés à la corrélation des items entre eux, le carré de la distance euclidienne a été utilisé pour mesurer la proximité des observations, comme préconisé par Hair et al (2010). Les analyses ont été menées selon la méthode d'Hair et al. (2010) : les classifications hiérarchiques ascendantes (méthode de Ward sur l'échantillon total puis sur 3 sous-échantillons aléatoires - respectivement de 94,93 et 93 individus) ont convergé vers une typologie en 5 groupes. Une classification non-hiérarchique (K-means) a été réalisée en fixant le nombre de groupes à 5. Afin de caractériser les 5 profils obtenus, les autres variables et caractéristiques sociodémographiques des répondants ont été utilisées comme variables muettes. Les résultats ont été interprétés au regard des tests de significativité (tableau 6). Dans un premier temps, toutes les variables ont été intégrées à titre de variables muettes. Dans un second temps, les variables non significatives comme la situation familiale et la CSP ont été retirées du tableau pour des raisons de lisibilité. Le tableau 6 ne restitue ainsi que les variables permettant de différencier les profils obtenus. 
Tableau 6 : Typologie en 5 profils

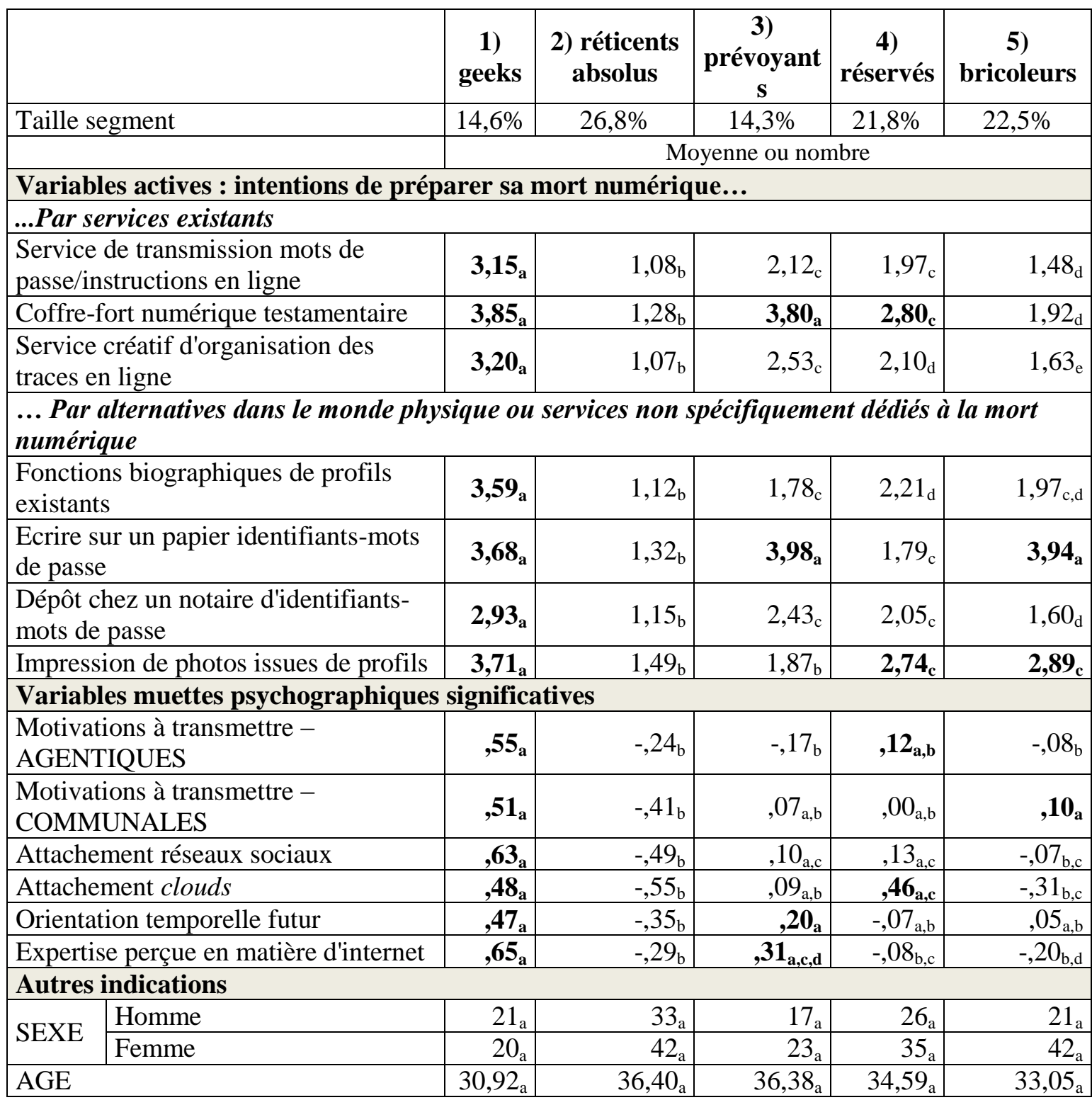

Remarque : les valeurs dans la même ligne et sous-table ne partageant pas le même indice diffèrent significativement à $\mathrm{p}<, 05$ dans le test bilatéral d'égalité pour proportions de colonne.

Profil 1 : Les geeks (14,6\% de l'échantillon). Ce groupe est constitué des individus les plus intéressés par les services liés à la mort numérique et ce, quels que soient les moyens utilisés. Ces individus ont les motivations agentiques et communales les plus fortes. Autrement dit, ils sont autant soucieux de l'image qui restera d'eux que préoccupés par le sort de ceux qui resteront. Cela s'explique par le fait que malgré leur jeune âge (30 ans en moyenne), ils pensent fortement au futur (orientation temporelle futur très forte). La seconde explication vient de leur degré d'attachement à leurs contenus numériques (réseaux sociaux et documents stockés sur des cloud). Ces jeunes, maîtrisant parfaitement Internet et les nouvelles technologies, créent des contenus numériques qui leur ressemblent et qui enferment une partie de leurs souvenirs. Dès lors, ils y sont attachés et s'interrogent sur leur conservation et transmission à travers le temps. 
Profil 2 : Les réticents absolus (26,8\% de l'échantillon). A l'opposé du groupe précédent, ce groupe rassemble les individus qui n'accordent aucun intérêt aux services de préparation à la mort numérique, qu'ils soient entièrement digitaux ou qu'ils incluent un passage par le monde physique. On peut penser que ces individus sont les plus réticents à envisager leur propre mort, d'autant plus qu'ils ne possèdent aucune des caractéristiques susceptibles de faire naître en eux un intérêt pour ces services. Pas du tout attachés à leurs profils de réseaux sociaux et encore moins au contenu de leurs clouds, ils se démarquent par le plus faible niveau de motivation à transmettre ces éléments numériques, que ce soit à des fins agentiques ou communales. Cela semble cohérent avec leur niveau d'expertise perçue et leur orientation temporelle. En effet, ils se déclarent les plus novices dans l'utilisation d'Internet et s'avèrent les moins orientés vers le futur.

Profil 3: Les prévoyants (14,3\% de l'échantillon). Si les individus qui composent ce groupe déclarent être peu attachés à leurs contenus numériques et peu enclins à les transmettre (les motivations agentiques sont très faibles), ils développent néanmoins une attitude positive à l'égard de la préparation à la mort numérique. Plus précisément, ces individus déclarent être intéressés par la souscription à un coffre-fort numérique testamentaire et ont l'intention d'écrire sur un papier leurs logins et mots de passe de comptes Internet, afin que leurs proches puissent y accéder en cas de décès. Ces résultats peuvent s'éclairer au regard de leur expertise perçue en matière d'internet et de leur orientation temporelle futur élevée. En effet, leur niveau d'expertise jugé moyen et leur tendance à se projeter dans l'avenir leur permettent de comprendre les enjeux futurs du web et la nécessité que pourraient avoir leurs proches d'accéder à certains documents et comptes en ligne après leur décès. Cependant, peu attachés à leurs contenus numériques, ils n'ont pas matière à en faire plus, en utilisant des services plus ludiques, relatifs à leurs photos, par exemple.

Profil 4 : Les réservés $(21,8 \%$ de l'échantillon). Ce groupe comprend des individus dont les intentions d'utiliser les services de préparation à la mort numérique demeurent légères, probablement parce qu'ils ne se projettent que très peu dans le futur (orientation temporelle futur neutre). Celles-ci apparaissent un peu plus prononcées en faveur du coffre-fort numérique testamentaire. Cela semble cohérent avec leur attachement, plus élevé pour les clouds que pour les réseaux sociaux. Ils sont ainsi habitués à déposer des documents dans des espaces virtuels et sont attachés à ces contenus. C'est ainsi via le biais de coffres-forts numériques que ces individus sont susceptibles de transformer leurs motivations, d'ordre agentique, en actions de transmission. Par ailleurs, l'action alternative la mieux accueillie est l'impression de photos, ce qui s'avère cohérent avec le léger attachement de ces personnes à leurs réseaux sociaux. Elles semblent ainsi attachées au contenu, notamment aux photos déposées sur leur profil, mais pas au profil en lui-même. Elles veulent ainsi conserver ces photos qui leur tiennent à cœur en les imprimant. Néanmoins, les actions ne semblent pas être envisagées dans l'immédiat. Elles pourraient se développer dans l'avenir. En effet, ce groupe est caractérisé par des motivations agentiques prononcées et plus élevées que les motivations communales.

Profil 5: Les bricoleurs (22,5\% de l'échantillon). Les individus composant ce segment sont peu attachés à leurs contenus numériques, mais développent néanmoins des motivations à les transmettre aux générations futures (motivations communales). Pour soutenir ces 
motivations, ils sont intéressés par les actions hors ligne, qui consistent à tangibiliser leurs contenus numériques (écrire sur un papier des identifiants et mots de passe, imprimer des photos issues de profils de réseaux sociaux). A l'image des prévoyants, ils semblent vouloir éviter à leurs proches d'éventuels soucis liés à l'accessibilité de leurs contenus numériques. Cependant, leur attachement à ces contenus et leur expertise d'Internet étant plus faible, ils se contentent de solutions simples, impliquant un passage par le monde physique, pour préserver l'essentiel. Une autre explication possible est qu'une partie de ces individus éprouve une méfiance à l'égard des sociétés qui commercialisent ces services et préfèrent développer des actions par leurs propres moyens.

\section{DISCUSSION DES RESULTATS}

Les problématiques liées à la mort numérique sont bel et bien perçues par les individus : seulement $26,8 \%$ des internautes (les réticents absolus) y sont hermétiques. Ce chiffre est impressionnant compte-tenu de la moyenne d'âge relativement basse des internautes. L'usage des services proposés n'en est qu'à ses balbutiements mais s'avère susceptible de se développer à l'avenir, à mesure que les générations actuelles (particulièrement les digital native) vont commencer à planifier leur mort. Cette partie discute des résultats d'un point de vue théorique, avant d'aborder les implications managériales de la recherche.

\section{Apports théoriques de la recherche et prolongements possibles}

Les résultats prolongent la littérature sur la préparation à la mort dans un contexte numérique. Les pratiques on-line semblent fonctionner comme celles concernant les biens tangibles. En effet, la typologie reflète le schéma classique de la préparation à la mort. Parmi les moins concernés, il y a ceux qui ne veulent pas s'en occuper - les réticents absolus - et ceux qui sont peu motivés mais se sentent obligés de s'y pencher, essentiellement pour des raisons communales (les prévoyants, les bricoleurs). Parmi les plus concernés, il y a ceux plutôt motivés pour des raisons agentiques (les réservés) et ceux, les plus intéressés, possédant à la fois des motivations agentiques et communales (les geeks). Le pourcentage d'individus appartenant à des profils susceptibles de prendre en charge la préparation de leur mort numérique ne signifie pas que le marché va nécessairement se développer. Comme dans le monde physique, les individus sont souvent réticents à aborder la question de leur propre mort et plus encore à la préparer.

Néanmoins, cette première approche descriptive nous offre un éclairage conceptuel concernant les relations entre les variables. La typologie permet de confirmer le rôle clé des variables comme l'orientation temporelle, l'attachement aux contenus numériques, les motivations à transmettre la mémoire et l'expertise perçue en matière d'internet, dans l'explication des intentions de préparer sa mort numérique. En effet, ce sont les seules variables muettes qui discriminent les profils (par opposition aux variables sociodémographiques et comportementales liées à l'activité actuelle en ligne, qui différaient peu entre les groupes et par conséquent étaient non-significatives). Les résultats semblent mettre en exergue le rôle médiateur des motivations des effets de l'attachement et de l'orientation temporelle sur les intentions. Autrement dit, les motivations à transmettre la mémoire résulteraient en partie d'un attachement aux objets reflétant la mémoire et d'une projection dans le futur. En effet, un score élevé/faible sur les deux variables entraîne des motivations fortes/faibles (respectivement chez les geeks et les réticents absolus), tandis que des scores opposés - par exemple un attachement fort et une orientation futur faible chez les réservés sont associés à des motivations modérées. De même, la typologie laisse envisager le caractère 
modérateur de l'expertise perçue sur le lien entre les motivations et les intentions. Cela est particulièrement saillant si l'on compare les prévoyants (expertise perçue forte) et les bricoleurs (expertise perçue faible), qui ont une structure de motivations similaire mais qui diffèrent dans le degré d'intention d'utiliser les services. Si cette typologie permet de faire émerger des relations causales entre les variables, une nouvelle collecte de données est nécessaire pour vérifier le pouvoir prédictif de cette modélisation.

\section{Voies possibles d'utilisation de la typologie pour développer les services}

Les résultats de cette recherche sont de nature à formuler des recommandations en matière de segmentation sur la base de critères psychographiques (motivations, attachement, rapport au temps) et de ciblage relatif aux services proposés. En effet, les cinq profils identifiés n'ont pas l'intention d'utiliser les mêmes services, d'où la nécessité de développer des arguments de communication variables selon les services et la cible visée. La première partie de l'article a mis en exergue deux catégories d'acteurs concernées par la problématique de la mort numérique et deux types d'arguments de communication.

Premièrement, les grands acteurs du web devant faire face au décès de leurs utilisateurs comme Facebook et Google ont pour objectif d'encourager leurs utilisateurs à anticiper le devenir de leurs contenus numériques déposés chez eux. La nature neutre du discours sur la démarche présentée comme une chose à faire doit faire sens auprès des prévoyants. Des arguments supplémentaires relatifs à la maîtrise de l'image (argument agentique) devraient satisfaire les geeks et les réservés. En outre, insister sur la simplicité d'utilisation et faire des tutoriels vidéo peut convaincre les réservés - caractérisés par une expertise moindre en matière d'Internet - à franchir le pas. Enfin, les bricoleurs peuvent être impliqués en leur indiquant explicitement la possibilité de se passer de la plateforme, en formulant leurs directives anticipées directement à leurs proches, dans une perspective davantage sociétale, à l'image des campagnes de communication pour le don d'organes.

Deuxièmement, les prestataires de services spécialisés dans la préparation de la mort numérique (ex: Edeneo, La vie d'après) ont pour objectif de vendre leurs services de préparation à la mort. Ils le font avec un discours sacré sur la mort insistant sur les arguments principalement agentiques (laisser une trace, maîtriser son image post-mortem) en accord avec les motivations des geeks et des réservés. Si ces prestataires rencontrent des difficultés pour se développer, c'est sans doute que ces arguments ne suffisent probablement pas à justifier le prix des prestations. L'apport de la recherche et de la typologie proposée est de mettre en évidence l'importance du degré d'attachement aux contenus numériques et de l'expertise perçue qui peuvent constituer des variables de segmentation. En ce sens, les variables d'ordre psychographique sont de nature à mieux comprendre les attentes des différents segments en matière de solutions de préparation à la mort numérique. Il est ainsi possible pour une entreprise de cibler un ou plusieurs profils en adaptant ses services et son discours (tableau 7).

\section{CONCLUSION}

D'un point de vue théorique, cet article prolonge la littérature sur la préparation à la mort dans un contexte numérique et identifie des concepts clés comme l'attachement aux contenus numériques, l'orientation temporelle et les motivations à transmettre la mémoire, favorisant l'intention de préparer sa mort numérique. Un prolongement consiste désormais à formuler et à tester des hypothèses relatives à l'articulation de ces variables au sein d'un modèle. D'un point de vue managérial, cet article permet de décrire les utilisateurs potentiels des services de 
préparation à la mort numérique et de fournir aux entreprises une typologie permettant de segmenter le marché pour adapter leurs offres de services. Des études qualitatives devront néanmoins être menées pour améliorer la compréhension de chacun des profils et aboutir à des campagnes de communication ciblées.

La recherche sur la mort numérique au sens large du terme n'étant qu'à ses balbutiements, cette recherche comporte quelques limites, essentiellement liées à sa nature exploratoire. Une première limite est relative aux intentions d'utiliser les services mesurées sans faire référence à un quelconque tarif. Dès lors, de futures recherches devront s'attarder sur la question du consentement à payer par les consommateurs. Sont-ils prêts à souscrire à des services payants? En effet, entretenir un profil sur un réseau social est gratuit pour le consommateur. Aussi, en ce qui concerne les cloud, seulement $11 \%$ des individus souscrivent à des formules payantes (étude Eurostat, 2014) . $^{5}$ Ainsi, un individu peut-il envisager de payer un service pour préparer le sort de ses contenus numériques, d'ores et déjà hébergés gratuitement ? A l'avenir, le consentement à payer semble déterminant pour savoir si une société se lançant sur le marché peut être rentable, ou si, à terme, ces services sont voués à devenir des services périphériques proposés par les sociétés du web, les notaires ou assureurs. Ces derniers restent pour l'heure relativement discrets sur la question de la mort en ligne.

Enfin, malgré un effort de diversification de notre échantillon (en termes de caractéristiques socio-démographiques et comportementales), il pourrait être intéressant de répliquer cette analyse sur d'autres échantillons. Ces futures recherches pourront intégrer d'autres variables sociodémographiques (le nombre d'enfants ou de petits-enfants par exemple) ou psychographiques comme la générativité. Elles pourront également s'interroger sur la nature des directives formulées (fermeture du compte, continuation, etc.) et recueillir le point de vue des futurs héritiers. Si un individu souhaite préparer sa mort numérique, est-il pour autant prêt à recevoir des instructions de la part de ses proches qui prépareraient également la leur?

\footnotetext{
${ }^{5}$ http://ec.europa.eu/eurostat/statistics-explained/index.php/Internet_and_cloud_services_-_statistics_on_the_use_by_individuals
} 
Tableau 7 : Des services et arguments de communication adaptés au profil ciblé

\begin{tabular}{|c|c|c|c|c|}
\hline Profils & $\begin{array}{c}\text { Critères de } \\
\text { segmentation }\end{array}$ & $\begin{array}{l}\text { Services } \\
\text { adaptés }\end{array}$ & $\begin{array}{l}\text { Acteurs } \\
\text { concernés }\end{array}$ & $\begin{array}{l}\text { Pistes à creuser pour } \\
\text { arguments de communication }\end{array}$ \\
\hline \multirow[t]{4}{*}{ 1. Les geeks } & $\begin{array}{l}\text { Attachement clouds } \\
\text { et RS : ++ }\end{array}$ & \multirow[t]{4}{*}{ Tous services } & \multirow[t]{4}{*}{ Tous acteurs } & \multirow{4}{*}{$\begin{array}{l}\text { Arguments fondés sur } \\
\text { l'attachement sentimental aux } \\
\text { contenus numériques couplés } \\
\text { aux motivations agentiques et } \\
\text { communales. }\end{array}$} \\
\hline & $\begin{array}{l}\text { Motivations } \\
\text { Agentiques } \\
\text { communales : ++ }\end{array}$ & & & \\
\hline & $\begin{array}{l}\text { Orientation futur : } \\
++\end{array}$ & & & \\
\hline & Expertise $:++$ & & & \\
\hline \multirow{4}{*}{$\begin{array}{l}\text { 2. Les réticents } \\
\text { absolus }\end{array}$} & Attachement -- & \multirow[t]{4}{*}{ Aucun service } & \multirow[t]{4}{*}{ Aucun acteur } & \multirow[t]{4}{*}{ Aucun argument. } \\
\hline & Motivations -- & & & \\
\hline & Orientation futur : -- & & & \\
\hline & Expertise -- & & & \\
\hline \multirow[t]{4}{*}{$\begin{array}{l}\text { 3. Les } \\
\text { prévoyants }\end{array}$} & $\begin{array}{l}\text { Attachement clouds } \\
\text { et RS : } \pm\end{array}$ & \multirow{4}{*}{$\begin{array}{l}\text { Service de dépôt } \\
\text { d'identifiants et } \\
\text { mots de passe en } \\
\text { ligne } \\
\text { Coffre-fort } \\
\text { numérique } \\
\text { testamentaire }\end{array}$} & \multirow[t]{4}{*}{$\begin{array}{l}\text { Institutionnels } \\
\text { et spécialistes }\end{array}$} & \multirow{4}{*}{$\begin{array}{l}\text { Arguments fondés sur } \\
\text { l'alternative aux mots de passe } \\
\text { éparpillés sur des papiers et } \\
\text { l'aide pour éviter les tracas } \\
\text { administratifs des héritiers. }\end{array}$} \\
\hline & $\begin{array}{l}\text { Motivations } \\
\text { communales : } \pm\end{array}$ & & & \\
\hline & $\begin{array}{l}\text { Orientation futur : } \\
++\end{array}$ & & & \\
\hline & Expertise $:+$ & & & \\
\hline \multirow[t]{4}{*}{ 4. Les réservés } & $\begin{array}{l}\text { Attachement : } \\
\mathrm{RS}(+) \text { et clouds } \\
(++)\end{array}$ & \multirow[t]{4}{*}{$\begin{array}{l}\text { Coffre-fort } \\
\text { numérique } \\
\text { testamentaire }\end{array}$} & \multirow[t]{4}{*}{ Spécialistes } & \multirow[t]{4}{*}{$\begin{array}{l}\text { Arguments fondés sur la } \\
\text { simplicité d'utilisation et le fait } \\
\text { de pouvoir laisser sa trace. }\end{array}$} \\
\hline & $\begin{array}{l}\text { Motivations } \\
\text { agentiques : }+\end{array}$ & & & \\
\hline & Orientation futur : \pm & & & \\
\hline & Expertise : - & & & \\
\hline \multirow{4}{*}{$\begin{array}{l}5 . \text { Les } \\
\text { bricoleurs }\end{array}$} & Attachement : - & \multirow{4}{*}{$\begin{array}{l}\text { Coffre-fort } \\
\text { traditionnel } \\
\text { Carnet de mots } \\
\text { de passe }\end{array}$} & \multirow{4}{*}{$\begin{array}{l}\text { Assurances, } \\
\text { banques, } \\
\text { notaire } \\
\text { (acteurs } \\
\text { traditionnels) } \\
\end{array}$} & \multirow{4}{*}{$\begin{array}{l}\text { Arguments fondés sur la } \\
\text { transmission de l'essentiel pour } \\
\text { les proches. }\end{array}$} \\
\hline & $\begin{array}{l}\text { Motivations } \\
\text { communales : ++ }\end{array}$ & & & \\
\hline & Orientation futur : \pm & & & \\
\hline & Expertise : -- & & & \\
\hline
\end{tabular}

\section{Bibliographie}

Bécheur A. et Gollety M. (2006). «Validation d'une échelle de mesure du Lead User », Revue Française du Marketing, vol. 1, $\mathrm{n}^{\circ}$ 5, 29-39.

Belk R. (2013). «Extended self in a digital world », Journal of Consumer Research, vol. 40, $\mathrm{n}^{\circ} 3,477-500$.

Carroll E. et Romano J. (2010). Your Digital Afterlife: When Facebook, Flickr and Twitter are your estate, What's Your Legacy? New Rider's Press, Berkeley, CA.

Curasi C., Price L. et Arnould E. (2004). « How individuals' cherished possessions become families' inalienable wealth », Journal of Consumer Research, vol. 31, n 3, 609-623.

Cushing A. (2012). Possessions and self extension in digital environments: Implications for maintaining personal information, $\mathrm{PhD}$ dissertation, University of North Carolina.

Denegri-Knott J., Watkins R. et Wood J. (2012). Transforming digital virtual goods into meaningful possessions, in Molesworth M. et Denegri-Knott J. (Eds), Digital Virtual Consumption, Routledge, London, 76-91.

Georges F. (2014). «Post mortem digital identities and new memorial uses of Facebook: Analysing the memorial page creators' identity », Thanatos, vol. 3, $\mathrm{n}^{\circ} 1,82-93$.

Guillemot S. et Gourmelen A. (2014). «Intergenerational transmission of digital data: user perceptions and the future », Actes du $1^{\text {er }}$ colloque Digital Intelligence, Nantes. 
Guillemot S. et Urien B. (2016). « Legacy writing and the consumption of biographic services», Psychology \& Marketing, vol.33, n¹1, 971-981.

Hair J., Black W., Babin B. et Anderson R. (2010). Multivariate Data Analysis, $7^{\text {ème }}$ édition, Prentice Hall.

Malas Z. (2012). Le rapport à l'avenir du consommateur âgé : entre épargne et consommation, In Guiot D. et Urien B. (eds), Le consommateur âgé, De Boeck, Bruxelles, 51-66.

Sohier R. et Brée J. (2016). «Clarification du concept d'identité digitale et proposition d'une échelle de mesure », Actes du 32ème congrès de l'association française du marketing, Lyon.

Urien B. (2003). «L'anxiété face à la mort et son influence sur le comportement du consommateur », Recherche et Applications en marketing, vol. 18, $\mathrm{n}^{\circ} 4,23-41$.

Urien B. et Guiot D. (2007). «Attitude face à la mort et comportement d'ajustement des consommateurs âgés : Vers l'élaboration d'une réponse marketing », Décisions Marketing, $\mathrm{n}^{\circ} 46,23-35$.

Usunier J.C. et Valette-Florence P. (2007). «The time styles scale. A review of developments and replications over 15 years », Time \& Society, vol.16, $n^{\circ} 2-3,333-366$.

Waagstein A. (2014). «An exploratory study of digital legacy among death aware people », Thanatos, vol. $3, \mathrm{n}^{\circ} 1,46-67$.

Zeller J. (2012). «Vers l'archivage total de soi ?», Actes des 12èmes journées des Archives de l'Université Catholique de Louvain, Belgique. 